Patient safety and prevention of unexpected events occurring during the intra-hospital

\section{transport of critically ill ICU patients}

\section{Dear Sir,}

We read with great interest the recent article on the intra-hospital transport (IHT) of critically ill patients by Venkategowda et al..$^{[1]}$ We agree with the authors that despite improvements in IHT practices, unexpected events (UE) incidents remain high. Since UEs can be reduced when an intensivist accompanies a critically ill patient, this practice should be incorporated routinely.

Epidemiological studies and feedback from intensive care societies have identified several risk factors related to patient, transport organization, technical, and human responsible for UE during IHT. ${ }^{[2]}$ These risks can be overcome by developing a culture of safety and standardization of procedures. Addressing the competence of the transport team is not the only factor that needs to be considered.

The first step starts even before ordering a diagnostic or therapeutic procedure, based on a benefit/risk analysis of IHT for a given patient. ${ }^{[3]}$ Additionally, preparation and management are also crucial steps when transporting critically ill patients. Having stabilized the critically ill patient before transport, technical, organizational, and human factors must be the subsequent targets for the primary prevention of IHT-related UEs. ${ }^{[2,4]}$

Overcoming the risks of IHT thus involves taking corrective action for all the causes at multiple levels and applying methods that have been proven to work in other sectors of activity. A more widespread use of checklists and proper training plans for teams are also expected to lead to an increase in IHT safety and a lowering of risks in the long term.
Kumari Shweta, Sachin Kumar Department of Hospital Administration, All India Institute of Medical Sciences (AlIMS), New Delhi, 'Department of Pulmonary Medicine, Institute of Liver and Biliary Sciences (ILBS), New Delhi, India

Correspondence: Dr. Sachin Kumar, C-15, Delhi Government Residential Complex, D-2 Pocket,Vasant Kunj, New Delhi - 110 070, India. E-mail: sachin.drk@gmail.com

\section{References}

1. Venkategowda PM, Mutkule DP, Taggu AN. Unexpected events occurring during the intra-hospital transport of critically ill ICU patients. Indian J Crit Care Med 2014;18:354-7.

2. Beckmann U, Gillies DM, Berenholtz SM, Wu AW, Pronovost P. Incidents relating to the intra-hospital transfer of critically ill patients. An analysis of the reports submitted to the Australian Incident Monitoring Study in Intensive Care. Intensive Care Med 2004;30:1579-85.

3. Caruana M, Culp K. Intrahospital transport of the critically ill adult: A research review and implications. Dimens Crit Care Nurs $1998 ; 17: 146-56$.

4. Fanara B, Manzon C, Barbot O, Desmettre T, Capellier G. Recommendations for the intra-hospital transport of critically ill patients. Crit Care 2010;14:R87. 